\title{
Remote Healthcare Monitoring and Assessment
}

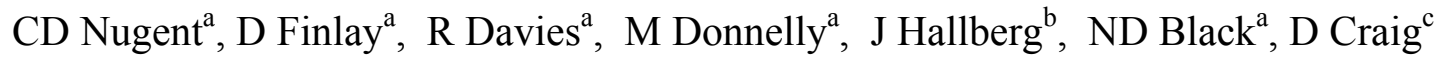

a: School of Computing and Mathematics, Faculty of Engineering, University of Ulster, Shore Road, Northern Ireland, BT37 0QB

b: Luleå University of Technology, Sweden

c: Belfast City Hospital/Queens University of Belfast, Northern Ireland

\section{Introduction to remote healthcare monitoring}

Remote healthcare monitoring is the process of assessing the well-being of a patient when the patient themselves and their healthcare professional are not physically together in the same room. Conventionally, patient assessment in Primary and Secondary healthcare provision involves a face-to-face consultation between the patient and the healthcare professional (General Practitioner, Consultant etc.). Advances in technology, specifically medical devices, sensors and high speed fixed and wireless communication networks have now made it possible to bring the assessment process to the patient, as opposed to limiting the assessment to the constraints of hospitals and doctors' surgeries. As a result, it is now possible for a patient to be assessed whilst in the comfort of their home or to have their vital signs monitored whilst, for example, shopping or at work. In addition, it is possible for patients to visit their local Doctor's surgery and benefit from expert consultants and receive their advice, without having to have a face-to-face meeting with them.

It is the aim of this review to introduce the concepts of remote healthcare monitoring, discuss the drivers which have lead to the realisation and uptake of this approach and to look at the benefits that evolving technologies can offer in this domain.

\subsection{Changes in Population Demographics}

We are at present witnessing a change in the way our population is represented, in terms of age profile. In the 1950s the largest percentage of the population was made up by those in the category of 0-9 years of age. At the turn of the century this category was amongst one of the largest, with a similar level of numbers being found in other age categories. Predictions for the next thirty years have shown that this trend will continue and infact result in a profile of age categories with almost similar sizes across all age groups (Figure 1). People are living longer and the total population size is increasing. In addition, the percentage of our population represented by those aged 65 and over is steadily increasing [1]. From a healthcare perspective this offers a number of challenges. In the first instance, we are faced by the simple fact that the population is now larger and hence places a larger demand on healthcare services. Secondly, there is an increased prevalence of healthcare problems and instances of disease within the elderly and thirdly there are now fewer younger people to care for the elderly [2].

Taking all of this into account has resulted in healthcare providers, governments and members of society, searching for new paradigms of healthcare delivery.

\subsection{The impact of Technology from social and economic perspectives}


We have previously introduced the concept that the increase in the size of the population has a direct impact on the prevalence of instances of healthcare problems and of long term chronic diseases. In addition to placing a resource burden on primary and secondary healthcare organisations, this increase in population size has additional societal and economic implications. There are benefits from both perspectives which would support the introduction of remote healthcare monitoring.

From a societal perspective, people are wishing to play a more active role in their own healthcare. This can be facilitated, at least notionally, via the realisation of remote healthcare monitoring where the patient is given a higher degree of responsibility to become more proactive with the management of their own healthcare and its assessment. Involvement in such a manner can also lead to the patient having a greater understanding of not only their condition, but also its means of assessment. In addition, there is the general appreciation that people recover more quickly in their own homes and would prefer to remain in their own homes, even if they are at greater risk [3]. Remaining at home for an extended period of time can also be linked to improvements in the patient's perceived quality of life. Taking this into account, the use of remote healthcare monitoring has the potential to avoid instances of institutionalisation when the patient requires high levels of healthcare assessment. It can also avoid lengthy and inconvenient trips being made for the purposes of routine clinical assessments. Patients can also benefit from remote expert clinical diagnosis. For example, it is possible to send a patient's clinical details from one institution to another and receive expert clinical assessment via remote means.

In addition to the societal benefits, deployment of technology to support healthcare assessment via remote means has a number of economic benefits. If a person can have their healthcare assessed within their own homes, removing the requirement of temporary or permanent institutionalisation, then significant healthcare savings can be gained. Additionally, those patients receiving clinical care within a hospital setting can be discharged in a much shorter space of time and continue to have routine assessments conducted via remote means. Finally, consultants can offer remote consultations to patients all over the world without having to leave their own office. This offers benefits to the patient in terms of them receiving treatment from 'world class' specialists who, because of geographical distances, would otherwise not be able to provide such treatment.

\subsection{Models of Patient-Healthcare Professional Interaction}

Traditionally, the means by which those involved in healthcare provision interact with their patients is based on a bi-lateral communication model (Figure 2 (a)). In this respect it is normal for the patient to have direct contact with a number of healthcare providers, however, commonly there is a potential lack of a unified infrastructure to support communication between healthcare providers. Deployment of technology and the establishment of care models facilitates communication channels to be established between both patients and healthcare providers as well as intercommunication between the healthcare providers (Figure 2 (b)). This offers an improved means of healthcare delivery from the patient's perspective as all those involved in the delivery of their care can interact. 
Although the aforementioned care models can be used to facilitate the deployment of technology, their conceptualisation can at times be faced with resistance. The deployment of technology within the realms of healthcare provision can offer benefits to the patients, however, the changes of practice which may be required from the healthcare providers' perspective, in addition to the problem of new service integration, creates major challenges concerning the widespread deployment of the technology. On the one hand, drivers such as the benefits of improved treatment results and alleviation of the problems associated with the shortage of physicians and nurses encourage such developments. Nevertheless, such innovations are faced with the constraints such as technology costs, reimbursement issues and legal issues, to name but a few. This inevitably results in a 'push-pull' scenario (Figure 3) from a technological perspective. The development of new technology results in new paradigms being pushed into the healthcare market. These can be considered as high risk solutions. If, on the other hand, a user driven approach is adopted, a pull effect can be witnessed. This may be viewed by some as the desired effect, where the end solution will be based on mature technology, which, from a technological perspective may not offer high levels of innovation, however, from the healthcare perspective can be seen as a low risk solution meeting the needs of the end users. In order to provide the patient with the best solution to their needs, a user centered design process offers many benefits. Adopting this approach allows the patient to be involved during the early stages of the design process.

\subsection{The impact of the Internet on Healthcare Delivery}

The Internet is one of the most widely used and accepted forms of modern day communication, as well as a rich source of information. Patients can now seek healthcare advice, research their symptoms and determine possible diagnosis, in addition to researching available courses of treatments. Additionally, the Internet can be used as a forum to support communities offering discussion groups dealing with health and healthcare topics. Furthermore, the Internet offers great potential for distributed healthcare, which is important especially for sparsely populated rural areas, and for people with limited mobility.

The Internet makes it possible for user-centric healthcare, where the user's needs are in focus. More demands are also placed on being able to locate the necessary information and help in user understanding. This has created more demands on information and communication resources, as well as on available healthcare professionals. Nevertheless, this also means that for many health-related issues, the user can receive help from centralized healthcare resources. For example, a parent concerned about a child's skin rash could be taught how to acquire high-resolution pictures of the affected area and subsequently forward such pictures to a centralized healthcare service. This approach would offer a fast and efficient means of receiving direct feedback regarding the condition of the skin, without the necessity of the parent and child having to travel to the hospital for a physical examination.

\subsection{Telemedicine}

This Section aims to introduce the notion of Telemedicine and how technology can be deployed to link healthcare professionals together in an effort to improve the quality of the healthcare delivery process. 


\subsection{What is Telemedicine?}

Telemedicine has received many definitions over the years. Most notably it can be described as the remote use of medical expertise at the point of need [4]. This results in the use of telecommunications to facilitate the sharing of medical knowledge and the delivery of healthcare over a distance [5]. With this approach medical expertise can be delivered to remote sites, rural areas or understaffed regions. Although Telecare may also be considered as a form of Telemedicine, for the purposes of this article we will treat these two topics individually and focus within this Section on the element of Telemedicine and the associated technology which fosters a collaborative working environment between medical experts.

\subsubsection{Telephony}

A rudimentary means of providing telemedicine is via telephone communications. This can be in the form of two specialists discussing a patient's diagnosis or prognosis over large distances or similarly can relate to a consultation between a patient and a specialist. Other examples include automated services which patients access, via the telephone, to provide details regarding their current state of health. An example of this type of system is an automated diabetes service which permits patients to verbally enter information regarding their condition via an automated telephone system [6]. Such a system facilitates the routine assessment of a patient's condition without the need for direct consultation thus alleviating pressure on healthcare services.

\subsubsection{Video Conferencing Systems}

Video Conferencing Systems have become of one the most popular platforms used to facilitate telemedicine and subsequently improve communications between healthcare professionals [7]. Such systems typically include the ability to send and receive video, audio, and text between one or more users. Additionally, some systems provide tools, such as shared whiteboards, which facilitate the communication of ideas and thoughts via informal means. The main purpose of using video conferencing systems in telemedicine is to provide a sense of presence through the provision of visual instructions, or demonstrations to patients, and to visually present or describe problems and symptoms to healthcare professionals.

Video conferencing provides a wide range of services that healthcare providers can avail of for example nurses can use video conferencing to make house-calls while keeping contact with the doctor, whilst patients can use the system to set up meetings with healthcare professionals from their home, instead of having to travel to the hospital.

\subsubsection{Point-to-Point systems}

Similar to video conferencing, point-to-point systems offer commutations between stakeholders, however, they only provide communications between two points. At present, several such systems are freely available. While these systems are often sufficient in providing simple communication between a patient and the doctor, they do not offer the support group functionality that video conferencing systems do. 


\subsubsection{Remote Surgery}

At the other end of the spectrum from telephony systems exists the concept of remote surgery. This technology provides the means to support the delivery of remote care through robotics [8]. An obvious advantage of this approach is that it permits world renowned surgeons from across the globe to provide patient care. The first remote surgery was conducted in 2001 and was referred to as "Operation Lindbergh". Through the use of three robotic arms setup in an operating theatre in France, surgeons located in New York where able to remotely perform a gallbladder operation over a fibre optic connection. Since this date, several other remote surgical procedures have taken place.

\subsubsection{Store and Forward Services}

In addition to real-time telemedicine systems such as those already described, offline telemedicine is also possible. Such care delivery does not require both parties to be present at the same time of communication. For example, technology within one consultant's office can be used to record some patient information which can be subsequently stored and forwarded at a set time to be reviewed offline by another consultant.

\subsection{Telecare}

This Section aims to introduce the notion of Telecare and how technology can be deployed to link remote patient information with healthcare providers in an effort to improve the quality and the healthcare delivery process.

\subsection{What is Telecare?}

Telecare combines the usage of sensing devices and telecommunications to support the remote monitoring of a patient within their home environment with the goal of providing a means of care support. Therefore, via remote patient monitoring it is possible for a Telecare service to react to abnormal situations which are a cause for concern. In response, a Telecare service can issue an alert to a caregiver or a family member. It is also possible for the person themselves to raise an alarm in instances when they may require support, for example, following a fall.

\subsubsection{Technology platforms available for home based monitoring}

To provide a home based monitoring system to patients who require additional health care services requires exploiting current technology platforms that are already in place. These should be widely available and practical in terms of cost, performance and speed.

The Public Switched Telephone Network (PSTN) is a commonly used means of supporting the transmission of information from the patient's home to a healthcare organisation. The PSTN is available throughout the world and although originally based on analogue lines, is now almost entirely digital at its core. The primary use of the PSTN is the ability to connect people together to provide a phone based voice service. However, in more recent times, the PSTN has been used as a digital 
communication channel allowing information to be transferred to and from patients' homes. The PSTN itself has a number of drawbacks in comparison with more recent technologies based on the same infrastructure. It can be more difficult to set up and is expensive to run if the application is resource intensive and operates at relatively slow speeds making it suitable for only a small percentage of applications.

Broadband technology is another possibility. As its name suggests, broadband technology offers a wider band of information. One example of broadband is a Digital Subscriber Line (DSL) which encodes digital information at a higher frequency on one channel and sends voice on a lower frequency forming another voice based channel. DSL technology offers high availability, as it is based upon the already existing PSTN system which has worldwide coverage. There are a number of advantages with broadband technology. Firstly, it is easy to set up and easy to use and can even support wireless connectivity. Although dependent on line conditions such as the distance from the exchange, the speed and reliability are greatly improved. Broadband technology, and DSL in particular, are essential to providing the underlying communication infrastructure to allow home based services to become successful.

\subsubsection{Telecare Services}

One of the most common forms of Telecare services exist in the form of alarm based pendants. Alarm based pendants are essentially a device worn by a patient which can be used in either a passive or active manner. Their primary use is within the home environment and can be used to trigger various alarms. A passive pendant is one which involves no interaction between the patient and the device; this could take the form of a fall detector which passively monitors the orientation of the patient during home based activities. Falls are common place among elderly patients living alone and are the leading cause for such people having to enter into permanent institutional care. Such a device could alert a relative or health care personnel to a serious situation. A more obvious and active approach of an alarm pendant is one that is activated by the patient whenever a security situation arises. Once the pendant has been activated, an emergency message is relayed to a healthcare professional or family member so that the necessary intervention can take place.

In addition to pendant alarms, Telecare services can be based upon a number of devices which may exist within the home environment, for example medication management devices, cognitive prosthetics, fire/smoke/water alarms and various door and device interaction sensors. The exchange of physiological data recorded from the patient in their home and transferred to a remote site can be referred to as Telehealth. Although the patient and the communication infrastructure for both Telecare and Telehealth are the same, it has been usual in the past to keep these two terms separate. Typical parameters which can be monitored within the auspices of Telehealth include blood pressure, electrocardiogram, weight and blood glucose levels.

\section{$4 \quad$ Ambient and Pervasive Computing}

The way in which healthcare can be delivered is changing rapidly. The convergence of mobile communications, decision support systems and Internet computing are 
offering a wealth of new healthcare paradigms. In this Section, we focus on a number of new and emerging technologies and demonstrate how they can be deployed within the realms of home based healthcare.

\subsection{Intelligent Homes}

Perhaps the most notable advance in care delivery has been through the introduction of the Intelligent Home environment [9]. This environment aims to promote the use of embedded technology to support a person within their own living environment. The desired goal is to offer a means of independent living and extend the period of time a person can remain in their own home prior to institutionalisation [2]. Within such an environment, it is common to find two types of devices; sensors and actuators. Sensors are the devices which can record information about the person in the environment. These may be motion sensors detecting which room the person is in, or they may be pressure sensors detecting whether the person is in, for example, a chair or their bed. Other types of sensors include temperature sensors, door sensors, water sensors, appliance sensors etc. Such sensors can provide sufficient information so that the current status of the person can be inferred and subsequently be reused to recommend how the environment should be modified or controlled. The environment itself is managed through the use of actuators. These can, for example, control the ambient temperature if the environment becomes too warm, or raise an alarm if a hazardous situation arises. For example, a person turns on the cooker and then turns on the taps in the bath. The challenge at present is to find the correct balance between the information gathered by the sensors and the ensuing processing to support the dynamic change of the environment through the use of the actuators to support the changing needs of the person.

\subsection{Wearable Systems and Smart Clothes}

The ability to monitor a number of vital signs from a patient in a pervasive manner has been realised through the introduction of Wearable Computing or Smart Clothes [10]. Smart clothing is a result of the consolidation of sensor technologies, advanced textiles and information and communication technologies [11]. Sensing devices can be embedded into clothing to offer a means of recording information such as heart rate, perspiration levels, body motion / movement and breathing rates. Smart clothing offers the ability to record from a larger surface area on the body, for example the torso, hence the limitations of taking recordings from the finger or the wrist can be avoided [12]. It is possible, during the manufacture of the garment, to fabricate the sensors by either coating a textile with a form of sensing material or to form a sensing material which can then be knitted or woven into the garment. Although many challenges now exist regarding which are the optimal places to locate the recording electrodes [13] and what information should be recorded, smart clothing provides a realistic solution to continuously monitor the person in an unconstrained manner and either provide a means of local processing or relay the information to a central location for assessment by healthcare staff. Smart clothing has huge potential in conjunction with the aforementioned intelligent environments as an augmented means, not only to assess the activities of the person, but also, their health status.

\subsection{Data Processing and Context Awareness}


We have described a number of paradigms whereby information relating to the patient can be shared between medical professionals or can be viewed remotely by a medical professional to offer a means of support. Given the ability to store large amounts of data about the person, their activities and their general health conditions through a number of vital sign markers, it has now become possible to consider deploying data processing systems with the ability to automatically understand the current status of the person. One of the biggest areas of interest at present is the ability to monitor changes in lifestyle and adapt the technology within the environment to support the changing requirements of the patient. As such, much effort is being directed towards the development of data processing systems which aim not only to detect trends within a person's behaviour but to try to understand the cause of such behaviour and attempt to correlate this with other social or environmental behaviours.

In addition to data processing, the introduction of Context Aware computing within healthcare and intelligent environments has also become more prevalent [14]. The term 'context' refers to any information which can be used to characterise the situation of a person or computational entity. Context aware computing is used to extract, interpret and then use contextual information in such a way that the system or environment can adapt its current state of operation to match the current context of its use.

\section{Case study: Home Based Medication Management}

We will now show how some of the aforementioned topics have been addressed in a real project scenario - Home Based Medication Management. Within this context we present an exemplar of how the problem domain has been identified, how a technical solution has been deployed and how emerging technologies have been integrated into the second generation of the system.

\subsection{Problem Domain}

It is a well recognised problem that patients do not fully adhere to their medication regimen having received a prescribed course of medication. This results in a significant burden being placed on the healthcare service due to extended patient welfare and healthcare costs. According to the World Health Organisation (WHO) adherence is defined as "the extent to which a person's behaviour - taking medication, following a diet, and/or executing lifestyle changes, corresponds with agreed recommendations from a healthcare provider" [15]. There have been numerous attempts to quantify the impact of non-adherence to medication, including: -one third of people take all their medication, one third take some and one third do not take any at all.

-as many as 125,000 deaths may occur each year in the US as a direct result of nonadherence to medication.

-up to $23 \%$ of admissions to nursing homes can be attributed to non-adherence.

Adherence to medication is a complex issue and stems from problems such as the patient not understanding their medication regimen, perceived side effects and financial issues [16]. These can also be further complicated by any confusion which may arise through the context in which the medication is prescribed, delivered and taken by the patient. 


\subsection{Potential deployment of technology}

Given the huge impact associated with non-adherence, many efforts have been made to deploy technology as a potential means to alleviate the effects of the problem [17]. Technology has been deployed in three different manners. In the first instance, pill holders can be offered to the patient which store the medication in labeled compartments for various times throughout the day. The second type of device is an extension of the first, however, electronic modules support the inclusion of preprogrammable alarms. The third is a monitoring device which provides a remote means to assess if the patient has taken their medication. This last type of device will be the focus of this Case Study.

\subsection{The MEDICATE system}

The aim of the MEDICATE system was to develop an Internet based care model and associated peripherals to support the needs of all stakeholders within the prescribe to intake chain of medication. The anticipated benefits of the system were the ability to improve and support the patient with the management of their medication in addition to providing a means to support communications between all stakeholders. To achieve this vision it was necessary to develop a suite of interfaces which could be used by each of the stakeholders. The interfaces took the form of both custom electronic devices and software interfaces all connected via an Internet based care model as shown in Figure 4.

This care model supported communication between the patient within their home and their remote formal or informal carer, in addition to a communication channel between healthcare professionals. Figure 5 below shows the various stakeholder interfaces. Briefly these include:

- Patient: a mobile medication management system (Figure 5 (a)) and a base station acting as a reservoir of medication and a means to connect the patient to the Internet portal. These devices had the ability to store the medication and would remind the patient at the appropriate time to take their medication. Any instances of non-adherence would be recorded by these devices

- Doctor: a web based interface (Figure 5 (b)) to support the prescribing of medication and assessment of patient adherence. This interface provides a means for the medication regime of the patient to be entered onto the system.

- Pharmacist: a software interface (Figure 5 (c)) to support the filling of medication containers to be used by the patient's medication device according to their prescribed medication regimen. This system has the ability to retrieve the information entered onto the system by the Doctor.

- Caregiver: this software interface (Figure $5(\mathrm{~d})$ ) provides a means to allow the patient's adherence to their medication regimen to be monitored in real time and, in instances of non-adherence, will raise the attention of the care giving staff.

Within this scenario we have demonstrated how the basic concepts of utilising modern day communications have supported the integration of a number of stakeholders in the supply-to-intake chain of medication. Evaluation of this system has demonstrated its usefulness in terms of supporting a patient's adherence to 
medication. Nevertheless, it has also highlighted the importance which should be given to the integration of such systems into existing practice.

The natural evolution of this system has involved the deployment of the concepts of medication management onto mobile phone based platforms [17]. These facilitate a similar means of providing management and of monitoring of a patient's intake of medication, in addition to maintaining the link with healthcare professionals. An additional extension to the system would involve integration with context aware services, where the means by which the reminder was offered to the patient would be dependent upon their current activity.

\subsection{Conclusions}

Overall, within this review we have shown how technology can be used to establish a number of care paradigms, all of which aim to improve the delivery of care provided to the patient and to overcome geographical boundaries. In some cases existing technological infrastructures may be used and in others new forms of technology are required. Prior to widespread deployment, a number of challenges still remain. These challenges are only partly related to the technology. Other challenges are more related to organizational issues and issues relating to who is actually going to pay for the technology and its associated services. Nevertheless, it is widely accepted that there are benefits to be accrued, hence it is anticipated that further effort will be directed towards these areas in future years. 


\section{Glossary}

PSTN Public Switched Telephone Network

DSL

Digital Subscriber Line

WHO

World Health Organisation 


\section{References}

[1] Ageing and life course, World Health Organisation, http://www.who.int/ageing/en/ (accessed May 2008).

[2] M.E. Pollack, Intelligent technology for an aging population: The use of ai to assist elders with cognitive impairment, AI Magazine, (2005), 9-24.

[3] D.J. Cook and S.K. Das, How smart are our environments? An updated look at the state of the art, Journal of Pervasive and Mobile Computing, 3 (2007), 5373.

[4] L. Belochi, Telemedicine Glossary: Glossary of Concepts, Technologies, Standards and Users, $5^{\text {th }}$ ed., European Commission, Brussels, 2003.

[5] H.R. Fischer, S. Reichlin, J.P. Gutzwiller, A. Dyson, C. Beglinger, Telemedicine as a new possibility to improve health care delivery, in M-Health: Emerging Mobile Health Systems, eds. RSH Istepanian, S Laxminarayan, CS Pattichis, (Springer, 2006), 203-218.

[6] L.A. Black, C. McMeel, M. McTear, N. Black, R. Harper, M. Lemon, Journal of Telemedicine and Telecare, 11 (2005), 6-8.

[7] M. Drugge, J. Hallberg, P. Parnes, K. Synnes, Wearable systems in nursing home care: prototyping experience, IEEE Pervasive computing, (2006), 86-91.

[8] J. Rosen and B. Hannaford, Doc at a distance, IEEE Spectrum, 43 (2006), 3439.

[9] J.C. Augusto and C.D. Nugent, Designing Smart Homes: The Role of Artificial Intelligence, LNAI 4008, Springer, 2006.

[10]F. Axisa, P.M. Schmitt, C. Gehin, G. Delhomme, E. McAdams and A. Dittmar, Flexible technologies and smart clothing for citizen medicine, home healthcare, and disease prevention, IEEE Transactions on Information Technology in Biomedicine, 9 (2005), 325-336.

[11]C.D. Nugent, P.J. McCullagh, E.T. McAdams and A. Lymberis, Personalised Health Management Systems: The Integration of Innovative Sesning, Textile, Information and Communication Technologies, (Amsterdam: IOS Press, 2005).

[12]S. Brady, L. Dunne, A. Lynch, B. Smyth and D. Diamond, Wearable Sensors? What is there to sense?, in Personalised Health Management Systems: The Integration of Innovative Sensing, Textile, Information and Communication Technologies, (Amsterdam: IOS Press, 2005), 80-88.

[13]M. Donnelly, C. Nugent, D. Finlay, P. McCullagh and N. Black, Making smart shirts smarter: Optimal electrode placement for cardiac assessment, International Journal of Assistive Robotics and Mechatronics, 8 (2007), 53-60.

[14]S.S. Intille, Designing a home of the future, IEEE Pervasive Computing, 1 (2002), 76-82.

[15]World Health Organisation (WHO), Adherence to Long-Term Therapies, Evidence for Action, 2003.

[16]L. Blonde, Removing polytherapy as a barrier to adherence, Managed Care, Compliance and Persistence with Medication Therapy, 9 (2000), 1.

[17]C.D. Nugent, D. Finlay, R. Davies, M. Mulvenna, J. Wallace, C. Paggetti, E. Tamburini, N. Black, The next generation of mobile medication management solutions, International Journal of Electronic Healthcare, 3 (2007), 7-31. 


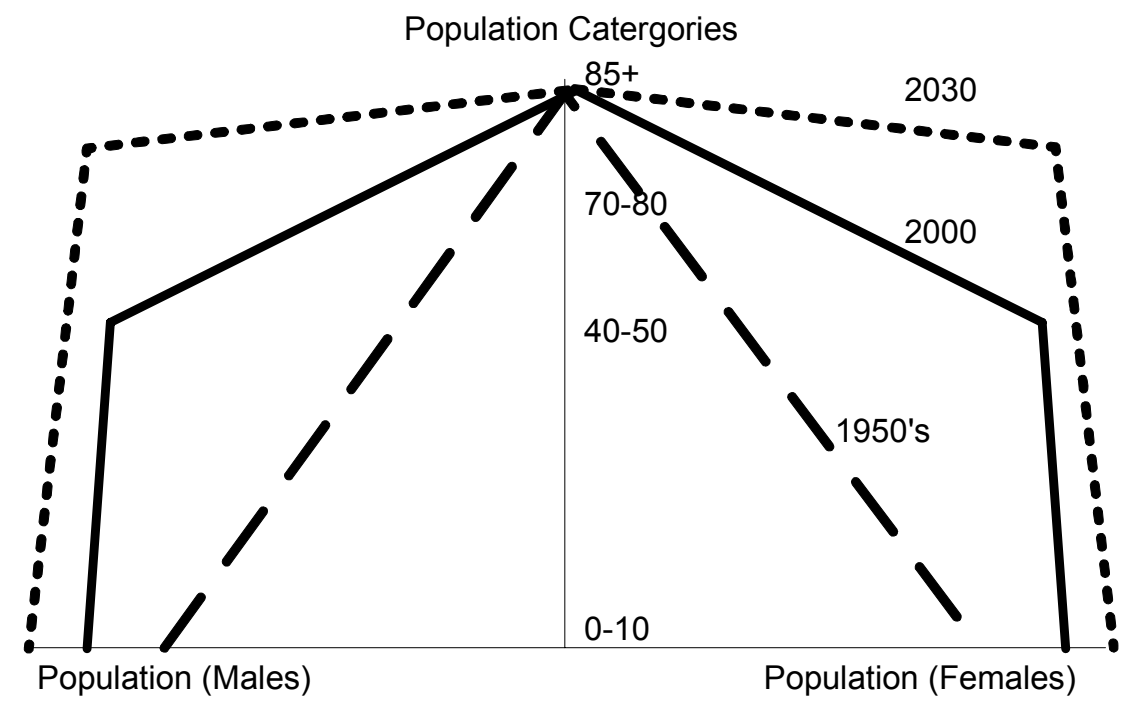

Figure 1 Estimated profile of age categories in 1950, 2000 and predictions for 2030. 


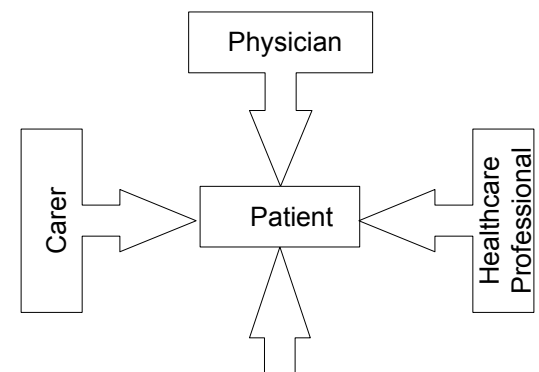

Pharmacist

(a) Interaction between stakeholders: the classic situation

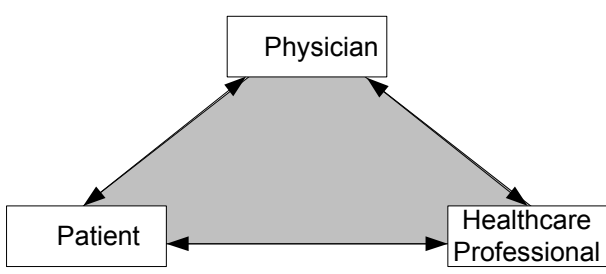

(b) Enhanced stakeholder

interaction facilitated via

technological communications

Figure 2 Examples of Patient-Healthcare Professional interactions. 


\section{Push Effect}

High risk enabling

new solutions

Pull Effect

Driven by user requirements and mature solutions

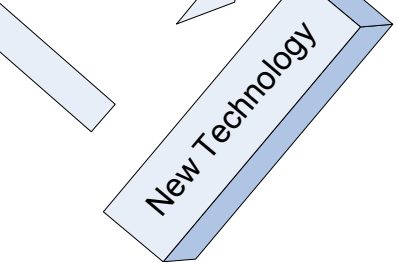

Figure 3 'Push-Pull' effect of technology within healthcare market place. 


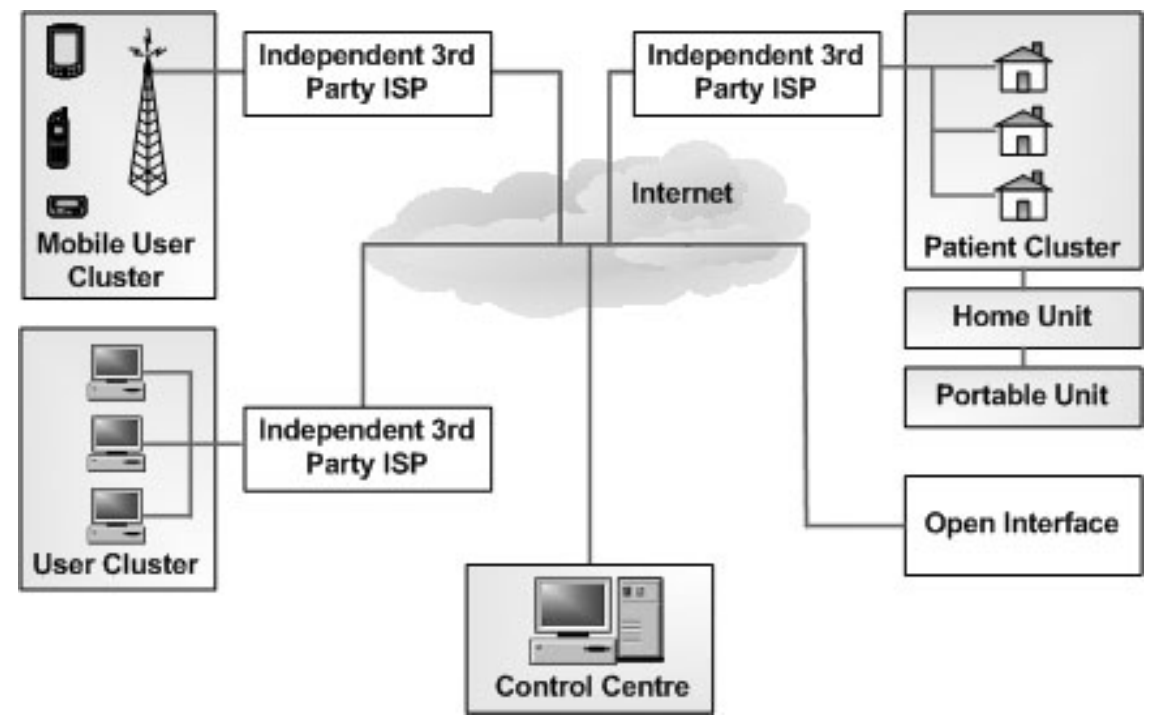

Figure 4 Overview of MEDICATE care model to support home based medication management. 


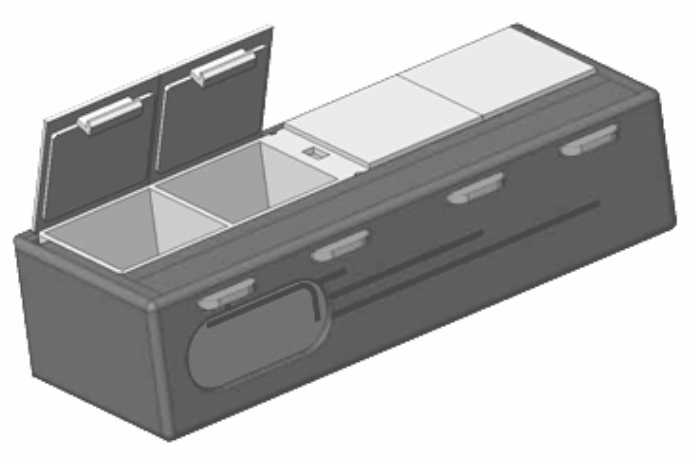

(a) Mobile medication device

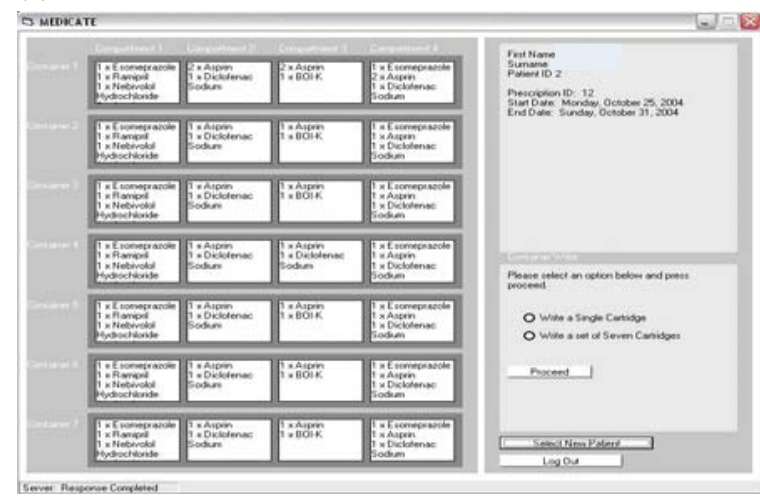

(c) Pharamacist's interface

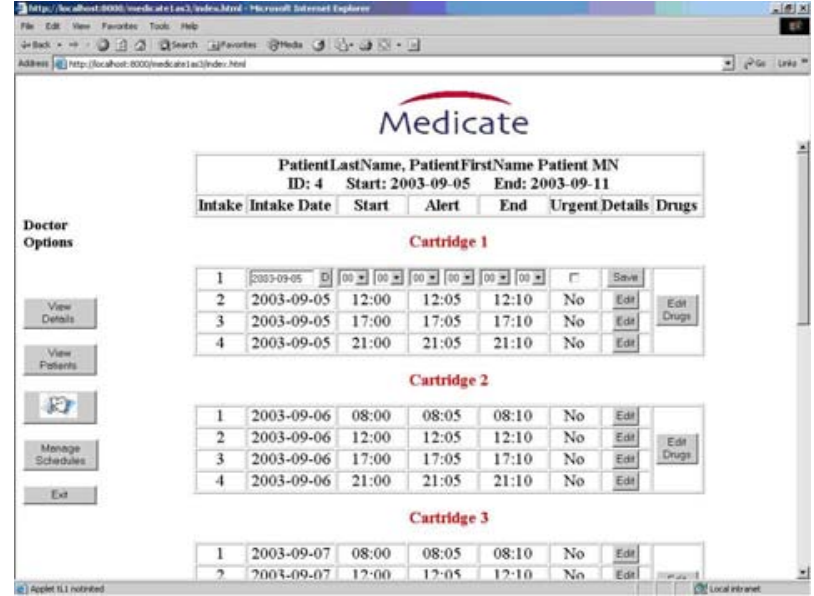

(b) Doctor's Interface

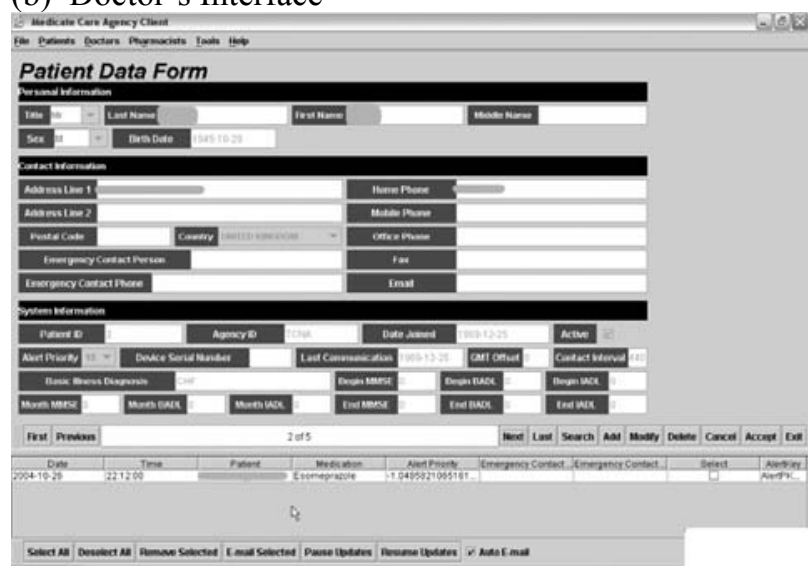

(d) Caregiver's interface

Figure 5 Stakeholder interfaces for the MEDICATE system. 